\title{
Power Spectrum of Discrete and Finite Series of Levels in Chaotic Resonators with and without Partially Violated Time-Reversal Invariance - The Case of Missing Levels
}

\author{
M. Bialous ${ }^{a, *}$ B. Dietz ${ }^{b}$ AND L. SiRKo $^{a}$ \\ ${ }^{a}$ Institute of Physics, Polish Academy of Sciences, \\ Aleja Lotników 32/46, 02-668 Warszawa, Poland \\ ${ }^{b}$ Lanzhou Center for Theoretical Physics and the Gansu Provincial Key Laboratory \\ of Theoretical Physics, Lanzhou University, Lanzhou, Gansu 730000, China \\ Doi: 10.12693/APhysPolA.140.545 \\ *e-mail: bialous@ifpan.edu.pl
}

\begin{abstract}
We report on experimental investigation of the power spectrum of discrete and finite series of levels in quantum billiards with chaotic classical dynamics and, respectively, with preserved and partially violated time-reversal $(\mathcal{T})$ invariance. In the experiment a flat microwave resonator with the shape of a two-dimensional quarter bow-tie billiard was used. The partial $\mathcal{T}$-invariance violation was induced by two embedded ferrites that were magnetized by an external magnetic field. Due to the openness and internal dissipation of the resonator and ferrites, one typically has to cope with incomplete sequences of resonance frequencies. We employ missing-level statistics to investigate the fluctuation properties in the spectra in terms of statistical measures such as the nearest-neighbor spacing distribution and the power spectrum. We confirm experimentally that the power spectrum can be directly used to evaluate the number of missing levels for both types of systems.
\end{abstract}

topics: wave chaos, microwave billiards, time-reversal invariance violation, chaotic scattering

\section{Introduction}

It has been conjectured that the fluctuation properties in the eigenvalue spectra of quantum systems, which are fully chaotic in the classical limit, are universal and described by random matrix theory (RMT) [1-4]. In this widely accepted and experimentally verified conjecture, the spectral properties of the associated Hamiltonian coincide with those of the Gaussian ensemble of random matrices belonging to the corresponding universality class, i.e., either orthogonal, unitary or the symplectic one. Examples for systems whose spectral properties are described by the Gaussian orthogonal ensemble (GOE, $\beta=1$ ) with preserved $\mathcal{T}$-invariance are flat microwave billiards [5-21], molecular spectra [22], atoms in strong microwave fields [23-35], quantum wells [36], and microwave networks [37-44]. The spectral properties of random matrices from the Gaussian unitary ensemble (GUE, $\beta=2$ ) have been observed in systems with $\mathcal{T}$-invariance violation (TIV), e.g., in nuclear reactions $[45,46]$, atoms in constant external magnetic fields [47], graphene quantum dots [48], Rydberg excitons [49], microwave billiards [50, 51] and networks [52-56]. Furthermore, the spectral properties of systems with partial TIV can be described by
RMT [21, 57-62]. In microwave billiards TIV is induced with magnetized ferrites, where the degree of time irreversibility can be adjusted, for example, by controlling the magnitude of the applied magnetic field and can be quantified by the parameter $\xi$ which changes from $\xi=0$ to $\xi=+\infty$ when the system undergoes a transition from $\mathcal{T}$-invariance to complete TIV. However, the transition from GOE to GUE already occurs for values of this parameter of the order $\xi \simeq 1[60,62-64]$. Recently, we found that the achievable size of TIV also depends on the openness $\eta$ of a chaotic system, i.e., the number of the scattering channels $M$ and the internal absorption $\gamma$. Its impact on the elastic enhancement factor $F_{M}(\eta, \gamma, \xi)$ was investigated for $2 \leq M \leq 9$ scattering channels in a microwave billiard containing magnetized ferrite $[63,64]$.

It was shown in $[53,65]$ that open quantum systems with violated $\mathcal{T}$-invariance symmetry can be conveniently studied using spectral correlation functions. In this type of studies it is indispensable to construct the cumulative level density $N\left(\nu_{i}\right)=$ $N_{\text {smooth }}\left(\nu_{i}\right)+N_{\text {fluc }}\left(\nu_{i}\right)$ of the identified resonance frequencies $\nu_{i}$, which is separated into a smooth part $N_{\text {smooth }}\left(\nu_{i}\right)$ and a fluctuating part $N_{\text {fluc }}\left(\nu_{i}\right)$. In order to analyze the statistical properties of chaotic systems, the energy levels need to be 
unfolded such that the level density is uniform the mean level spacing is constant, which is achieved by the replacement $\epsilon_{i}=N_{\text {smooth }}\left(\nu_{i}\right)$, implying that the mean spacing equals unity, i.e., $\left\langle\epsilon_{i+1}-\epsilon_{i}\right\rangle=1$. Especially in the case of the power spectrum analysis, the deviation of the $q$-th nearest-neighbor spacing from its mean value $q$ is considered $[53,65]$. The deviation is denoted as $\delta_{q}=\epsilon_{q+1}-\epsilon_{1}-q$. Typically, the completeness of the spectra is indispensable in the statistical analysis of the spectral properties of quantum and wave systems. However, this is rarely accomplishable in experiments. Examples for such an unusual situation are highprecision experiments performed with flat superconducting microwave resonators at liquid-helium temperature [21, 60]. However, for wave systems investigated at room temperature, due to absorption and openness, the appearance of incomplete spectra which implies deviations of their spectral statistics from the random-matrix theory predictions, is common. To overcome these difficulties, one has to properly deal with missing levels, which is possible by employing the missing levels statistics [53, 62] introduced in [66].

The objective of this article is to analyze the power spectrum statistics $[53,65]$ of real open systems with internal absorption that leads to incompleteness of the spectra. We use a flat microwave resonator with the shape of a quarter bow-tie to simulate a quantum billiard with preserved and partially violated $\mathcal{T}$-invariance. In the analysis we will consider both the strength of TIV expressed by the parameter $\xi[62,64]$ and the fraction of the observed levels $\varphi$.

\section{Experimental setup}

A schematic view of the microwave cavity is shown in Fig. 1. It simulates a two-dimensional quantum billiard of corresponding shape [63, 64]. The cavity with an area $A=1828.5 \pm 5.0 \mathrm{~cm}^{2}$ and a perimeter $L=202.3 \pm 2.0 \mathrm{~cm}$ was made of two plates of polished aluminum type EN 5754 . The cavity body was milled out of the bottom plate. The cut-off frequency equals $\nu_{\max }=c / 2 h \simeq$ $12.49 \mathrm{GHz}$, where $c$ is the speed of light in vacuum, and $h=1.2 \mathrm{~cm}$ is the height of the cavity. Below $\nu_{\max }$ only the transverse magnetic modes are excited inside the cavity so that the Helmholtz equation describing the microwave cavity is mathematically equivalent to two dimensional Schrödinger equation for a free particle in a quantum billiard of corresponding shape and both are governed by the same eigenvalue equation. The inner surface of the cavity was covered with a $20 \mu \mathrm{m}$ layer of silver to reduce the internal absorption. A metallic perturber $P$ with perimeter $L \simeq 26 \mathrm{~cm}$ and area $A \simeq 9 \mathrm{~cm}^{2}$ was placed inside the cavity and moved along the walls using a small cylindrical magnet in order to create different realizations of the cavity.

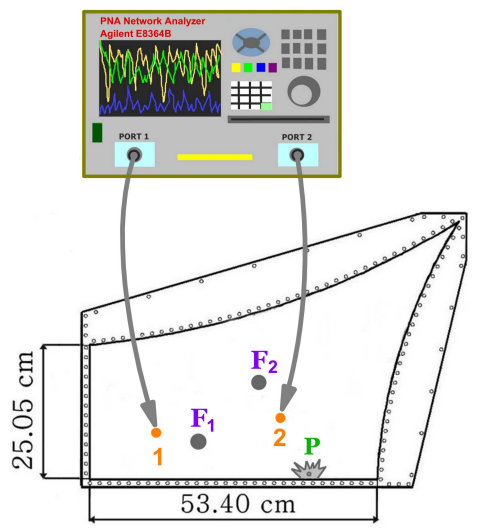

Fig. 1. Experimental setup for the measurement of the two-port scattering matrix $\hat{S}$ of the microwave billiard. The vector network analyzer Agilent E8364B was connected through flexible microwave cables to two antennas that were attached to the cavity at the positions marked by 1 and 2 . In order to induce $\mathcal{T}$-invariance violation two ferrites were placed inside the cavity between pairs of external magnets above and below the cavity at the positions marked by $F_{1}$ and $F_{2}$. Different realizations of the billiard were realized by moving a metallic perturber $P$ inside the cavity alongside its walls.

In order to induce partial TIV two cylindrical NiZn ferrites with a diameter $d=33 \mathrm{~mm}$ and height $h=6 \mathrm{~mm}$ and the saturation magnetization 2600 Oe (manufactured by SAMWHA, South Korea) were positioned inside the cavity at the locations marked by $F_{1}$ and $F_{2}$ in Fig. 1 . They were placed between two pairs of NdFeB magnets of diameter $d=33 \mathrm{~mm}$ and height $h=30 \mathrm{~mm}$ of the type N42 with coercivity 11850 Oe $(943 \mathrm{kA} / \mathrm{m})$ below the bottom wall and above the top wall of the cavity, respectively. The homogenous magnetic field of the strength $B \simeq 495 \mathrm{mT}$ induces macroscopic magnetization $M$ of the ferrites across their crosssections. The precession of magnetization around $B$ with the Larmor frequency $\omega_{o}=\gamma B$, where $\gamma=32.2 \mathrm{GHz} / \mathrm{T}$ denotes the gyromagnetic ratio, leads to the appearance of a ferromagnetic resonances at $\nu_{f r}=15.9 \mathrm{GHz}$.

In our analysis of the fluctuation properties of the unfolded resonances we consider only $M=2$ scattering channels since, as shown in [63], they lead to the strongest achievable TIV. The larger the number of scattering channels for a given microwave frequency, the weaker is TIV [63]. Furthermore, the openness and internal absorption for $M>2$ channels lead to stronger overlapping of resonances, rendering impossible the identification the corresponding resonance frequencies. To measure the two-port scattering matrix $\hat{S}(\nu)$ two antennas with length $5.8 \mathrm{~mm}$ and pin diameter $0.9 \mathrm{~mm}$ were attached to the resonator at the positions marked by 1 and 2 in Fig. 1 and connected to an Agilent E8364B Vector Network Analyzer (VNA). They act 
as scattering equivalent channels coupled to VNA. For systems with preserved $\mathcal{T}$-invariance the scattering matrix $\hat{S}$ is symmetric, i.e., transmission from antenna 2 to antenna 1 equals transmission from antenna 1 to antenna $S_{12}(\nu)=S_{21}(\nu)$. The closer, the microwave frequency is to $\nu_{f r}$ in the system with magnetized ferritse, the stronger is TIV, thereby leading to differing matrix elements $S_{12}(\nu)$ and $S_{21}(\nu)$.

Examples of transmission spectra from antenna 1 to antenna 2 and vice versa are shown in Fig. 2. The moduli of the scattering matrix elements $\left|S_{12}(\nu)\right|$ (black full line) and $\left|S_{21}(\nu)\right|$ (orange dotted line) are measured in the frequency range 8-9 $\mathrm{GHz}$. The ferrite properties are strongly frequency dependent and in this frequency the strongest TIV with $\xi=0.49$ is attained. Transmission spectra are displayed for the microwave billiard with preserved $\mathcal{T}$-invariance $(\xi=0)$ in Fig. 2a. In this case we expect reciprocity of the scattering matrix $\hat{S}(\nu)$, that is $\left|S_{12}(\nu)\right|=\left|S_{21}(\nu)\right|$ which is clearly demonstrated. In Fig. 2b, the spectra of the microwave billiard with partially violated time-reversal invariance are shown. In this case reciprocity does not hold, so that the scattering matrix $\hat{S}(\nu)$ is not symmetric $\left|S_{12}(\nu)\right| \neq\left|S_{21}(\nu)\right|$, which is confirmed.

In order to quantify the size of TIV, we use the cross-correlation coefficient $C^{\text {cross }}=$ $C^{\operatorname{cross}}(\eta, \gamma, \xi)[62]$

$$
C^{\text {cross }}=\frac{\Re\left[\left\langle S_{12}^{f l}(\nu) S_{21}^{f l *}(\nu)\right\rangle\right]}{\sqrt{\langle|\left(\left.S_{12}^{f l}(\nu)\right|^{2}\right\rangle\langle|\left(\left.S_{21}^{f l}(\nu)\right|^{2}\right\rangle}},
$$

where $S_{12}^{f l}(\nu)=S_{12}(\nu)-\left\langle S_{12}(\nu)\right\rangle$ denotes the fluctuating part of the scattering matrix element $S_{12}(\nu)$. For systems with completely violated $\mathcal{T}$-invariance the cross-correlation coefficient vanishes $C^{\text {cross }}=0$, because $S_{12}(\nu)$ and $S_{21}^{*}(\nu)$ are uncorrelated, and $C^{\text {cross }}=1$ for $\mathcal{T}$-invariant systems.

\section{Statistical fluctuations of the energy levels}

The transition between the orthogonal and unitary symmetry classes, GOE and GUE, induced by applying an external magnetic field $B$ was verified on the basis of the long-range correlation function - the power spectrum $\langle s(\tilde{k})\rangle[53]$. The quantity $\langle s(\tilde{k})\rangle$ is more sensitive to missing levels than the nearest-neighbor spacing distribution (NNSD) [53, 62]. Indeed, we will show that it can be used to determine the fraction of the observed levels $\varphi$ in the experiment.

The power spectrum $\langle s(\tilde{k})\rangle$ of a discrete and finite series $\delta_{q}=\epsilon_{q+1}-\epsilon_{1}-q$ for a sequence of $N$ levels is given by $S(k)=\left|\tilde{\delta}_{k}\right|^{2}$, where $\tilde{\delta}_{k}$ is the Fourier transform of $\delta_{q}$ of the form

$$
\tilde{\delta}_{k}=\frac{1}{\sqrt{N}} \sum_{q=0}^{N-1} \delta_{q} \exp \left(-\frac{2 \mathrm{i} \pi k q}{N}\right) .
$$

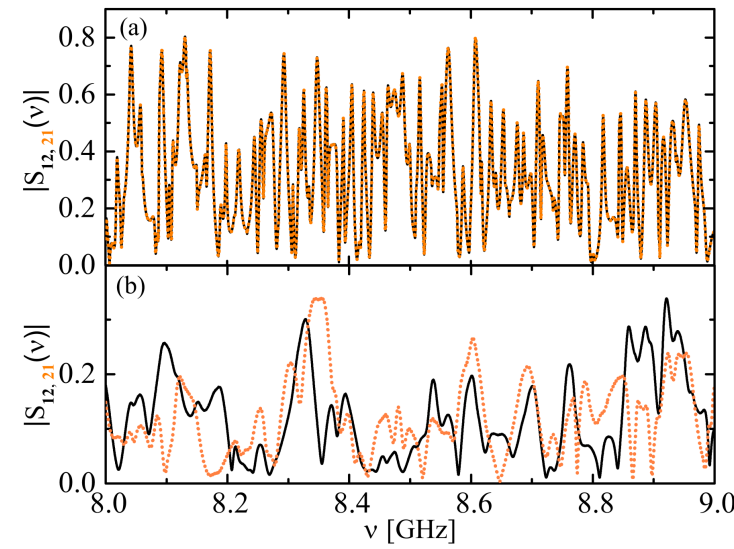

Fig. 2. Examples of transmission spectra. (a) Moduli of the scattering matrix elements $\left|S_{12}(\nu)\right|$ (black full line) from antenna 2 to antenna 1 and vice versa, $\left|S_{21}(\nu)\right|$ (orange dotted line), in the frequency range $8-9 \mathrm{GHz}$ for the microwave billiard with preserved $\mathcal{T}$-invariance $(\xi=0)$. (b) The same for the microwave billiard with partially violated time-reversal invariance $(\xi=0.49)$.

It was shown in $[68,69]$ that for $\tilde{k}=k / N \ll 1$ the power spectrum exhibits a power law dependence $\langle S(\tilde{k})\rangle \propto \tilde{k}^{-\alpha}$. For chaotic systems $\alpha=1$, independently of whether time-reversal invariance is preserved or not, and $\alpha=2$ for regular systems. Considering the fraction of identified levels $\varphi$ and the strength $\xi$ of TIV, the power spectrum $\langle s(\tilde{k})\rangle$ is expressed by

$$
\begin{aligned}
& \langle s(\tilde{k})\rangle=\frac{1}{4 \sin ^{2}(\pi \tilde{k})}-\frac{\varphi^{2}}{12} . \\
& +\frac{\varphi}{4 \pi^{2}}\left[\frac{K^{\xi}(\varphi \tilde{k})-1}{\tilde{k}^{2}}+\frac{K^{\xi}(\varphi(1-\tilde{k}))-1}{(1-\tilde{k})^{2}}\right] .
\end{aligned}
$$

Here, $0 \leq \tilde{k} \leq 1$ and $K^{\xi}(\tau)$ is the spectral form factor which is known analytically for $\mathcal{T}$-invariant systems and for the case of complete TIV. For the GOE, $K^{\xi=0}(\tau)=2 \tau-\tau \log (1+2 \tau)$ for $\tau \leq 1$, while for the GUE, $K^{\xi \rightarrow \infty}(\tau)=\tau$. For systems with partially violated time-reversal invariance $(\xi \neq 0)$ the spectral form factor $K^{\xi}(\tau)$ has been obtained from the analytical result for the two-point cluster function [57, 62].

For the system with partially violated $\mathcal{T}$-invariance, we analyzed the frequency range $8-9 \mathrm{GHz}$ in which maximal TIV is achieved. We took into account 30 randomly selected realizations of the cavity with, respectively, 90 eigenvalues. Thereby an ensemble of 2700 resonance frequencies was obtained. For the cavity with preserved time-reversal $\mathcal{T}$-invariance $(\xi=0)$, measurements were done in the frequency range $\nu=8-10 \mathrm{GHz}$. We took into account 15 randomly selected realizations of the cavity, having respectively $N=180$ eigenvalues in each sequence, which gave also 2700 resonance frequencies. 

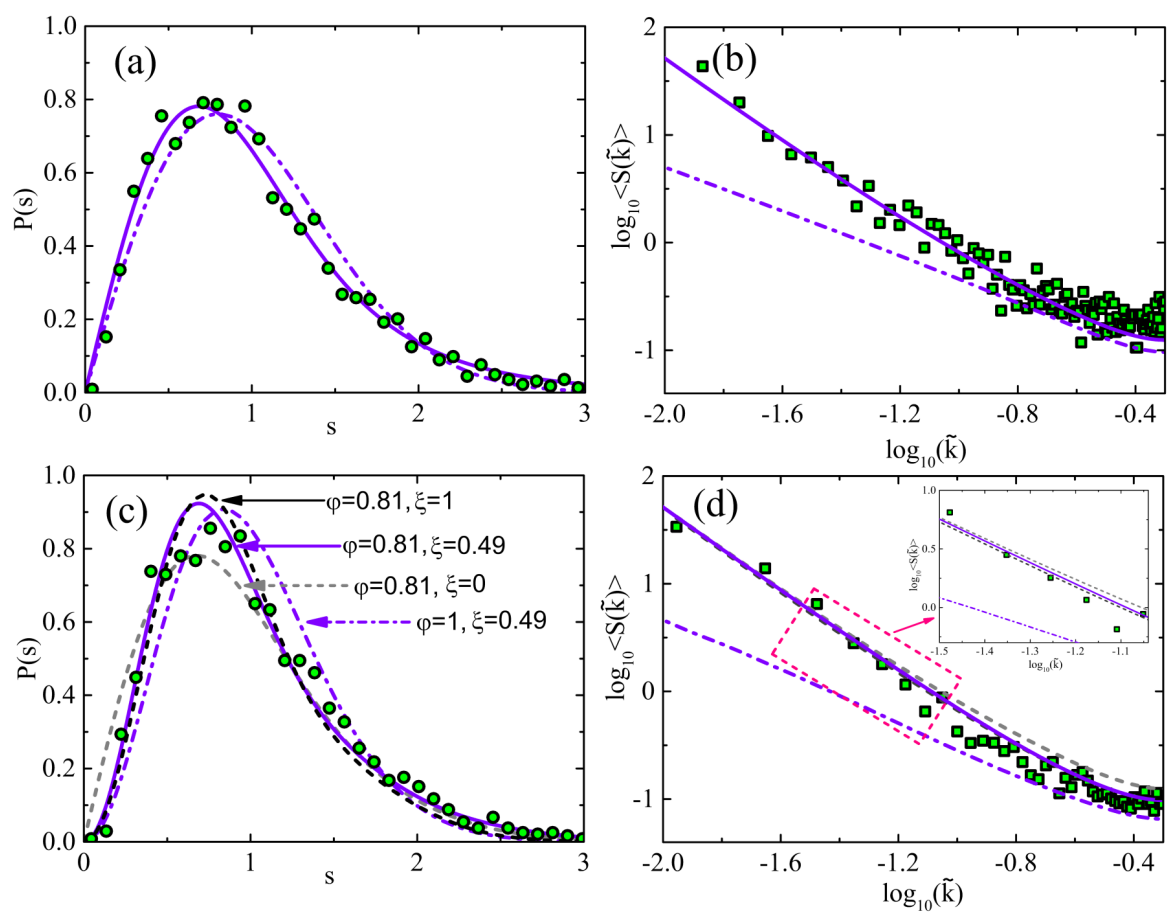

Fig. 3. Short- and long-range correlations of the unfolded eigenfrequencies. Panels (a, b) The nearest-neighbor spacing distribution (circles) and the power spectrum $\langle s(\tilde{k})\rangle$ (squares) for the microwave billiard with preserved $\mathcal{T}$-invariance $(\xi=0)$ and a fraction of observed levels $\varphi=0.81 \pm 0.03$. The experimental results in panels $(\mathrm{a}, \mathrm{b})$ are compared to those of the eigenvalues of RMT from the GOE (violet dash-dotted line) and to the theoretical predictions for $\varphi=0.81$ (violet full line). Panels (c,d) The nearest neighbor spacing distribution (circles) and the power spectrum $\langle s(\tilde{k})\rangle$ (squares) for the microwave billiard with partially violated time-reversal invariance $(\xi=0.49 \pm 0.02)$ and $\varphi=0.81 \pm 0.03$. The experimental results in panels (c, d) are compared to the theoretical ones obtained for the systems with partially violated time-reversal $\mathcal{T}$-invariance $(\xi=0.49)$ with the same fraction of observed levels $\varphi=0.81$ (violet full line). Additionally, the experimental results are compared to the missing level statistics for $\operatorname{GOE}(\varphi=0.81, \xi=0$, gray broken line) and GUE $(\varphi=0.81, \xi=1$, black broken line), respectively, as well as to the theoretical predictions for $\varphi=1.0$ and $\xi=0.49$ (violet dash-dotted line). The inset in Panel (d) shows the power spectrum in a narrower range of the parameter $\tilde{k}:-1.5 \leq \log _{10}(\tilde{k}) \leq-1.04$.

In Fig. 3, we compare the experimental results for short- and long-range correlations of the unfolded eigenvalues for the systems with preserved and violated $\mathcal{T}$-invariance. In Fig. $3 \mathrm{a}$ and $\mathrm{b}$ the nearest-neighbor spacing distribution (circles) and the power spectrum $\langle s(\tilde{k})\rangle$ (squares) are depicted for the microwave billiard with preserved $\mathcal{T}$-invariance $(\xi=0)$ for a fraction $\varphi=0.81 \pm 0.03$ of observed levels $\varphi=0.81 \pm 0.03$. The experimental results are compared to the ones obtained for GOE in the framework of RMT (violet full lines) and to the theoretical predictions for $\varphi=0.81$ (violet broken lines). In both cases, the experimental results are in good agreement with the theoretical ones. In Fig. 3c and d the nearest neighbor spacing distribution (circles) and the power spectrum $\langle s(\tilde{k})\rangle$ (squares) for the microwave billiard with partially violated time-reversal invariance $(\xi=0.49 \pm 0.02)$ and $\varphi=0.81 \pm 0.03$ are exhibited. The experimental results are compared to the theoretical ones [62] for $\xi=0.49$ and the same fraction of observed levels $\varphi=0.81$ (violet full lines), to the RMT results for
$\operatorname{GOE}(\xi=0)$ with $\varphi=0.81$ (gray broken lines), the $\operatorname{GUE}(\xi=1)$ with $\varphi=0.81$ (black broken lines) and to the theoretical predictions for $\xi=0.49$ and $\varphi=1$ (violet dash-dotted lines). Again, the experimental results are in good agreement with the theoretical ones.

One should point out that the case of partially violated $\mathcal{T}$ invariance is more involved than the case of preserved $\mathcal{T}$ invariance because the spectral properties depend om the fraction of observed levels $\varphi$ and on the strength of TIV quantified by $\xi$. However, the comparison of Fig. 3b and Fig. 3d clearly demontrates that for small values of $\log _{10}(\tilde{k})<-1.4$ the power spectrum is almost indistinguishable for the GOE and GUE, that is, it depends only marginally on $\xi$, but on the contrary it is extremely sensitive to the fraction of observed levels $\varphi$. The inset in Fig. 3d shows the details of the power spectrum in a narrower range of the parameter $\tilde{k}$ for $-1.5 \leq \log _{10}(\tilde{k}) \leq-1.04$. It clearly demonstrates strong sensitivity of $\langle s(\tilde{k})\rangle$ on $\varphi$ and a large deviation of $\langle s(\tilde{k})\rangle$ for $\varphi=0.81$ and $\xi=0.49$ (violet full 
line) from the one with $\varphi=1$ and $\xi=0.49$ (violet dash-dotted line). The weak dependence of $\langle s(\tilde{k})\rangle$ on $\xi$ for $\varphi<1$ makes it an exceptionally useful spectral tool for the experimental identification of the fraction of missing levels $(1-\varphi)$ for, actually, all fundamental symmetry classes as well as the ones with broken symmetries. Figure $3 \mathrm{a}$ and $\mathrm{c}$ show that the nearest-neighbor spacing distribution, on the contrary, depends rather weakly on both parameters $\xi$ and $\varphi$. Actually, in order to determine the value of $\xi$ from the spectral properties, one would need to consider the number variance which, however, relies on an ensemble of high statistical relevance [62].

\section{Conclusion}

We investigated the fluctuations of the resonances in a fully chaotic quarter bow-tie microwave billiard simulating quantum billiards with preserved time-reversal invariance and the ones with partial $\mathcal{T}$-invariance violation. The nearest-neighbor spacing distribution and the power spectra were considered as statistical measures for the spectral properties of both systems for $\nu=8-10 \mathrm{GHz}$ and $\nu=8-9 \mathrm{GHz}$, respectively. The ferromagnetic resonances were induced due to the presence of magnetized ferrites inside the resonators. In our analysis for $M=2$ open equivalent channels, we take into account the strength of TIV in terms of $\xi$ in combination with the incompleteness of the spectra expressed by the ratio of the observed levels $\varphi$. We found out that the power spectrum $\langle s(\tilde{k})\rangle$ indicates precisely the fraction of observed levels $\varphi$. Furthermore, the theoretical RMT analysis, which took into account both parameters $\varphi$ and $\xi$, reproduced very well the experimental results. It was demonstrated that the power spectrum can be used as a useful spectral tool for finding experimentally the fraction of the missing levels for systems belonging to the orthogonal and unitary universality class and for the systems undergoing the transition from GOE to GUE.

\section{Acknowledgments}

This work was supported in part by the $\mathrm{Na}$ tional Science Center, Poland, Grant No. UMO2018/30/Q/ST2/00324. B.D. thanks the National Natural Science Foundation of China for financial support through Grants No. 11775100, No. 1196113100, and No. 12047501. Supported by the 111 Project under Grant No. B20063.

\section{References}

[1] M. Berry, Structural Stability in Physics, Springer-Verlag, Berlin, 1979.

[2] G. Casati, F. Valz-Gris, I. Guarnieri, Lett. Nuovo Cimento 28, 279 (1980).

[3] O. Bohigas, M.J. Giannoni, C. Schmit, Phys. Rev. Lett. 52, 1 (1984).
[4] M.L. Mehta, Random Matrices, 3rd ed., Elsevier, Amsterdam 2004.

[5] B. Dietz, T. Friedrich, H. L. Harney, M. Miski-Oglu, A. Richter, F. Schäfer, H.A. Weidenmüller, Phys. Rev. E 81, 036205 (2010).

[6] J.-H. Yeh, Z. Drikas, J. Gil Gil, S. Hong, B.T. Taddese, E. Ott, T.M. Antonsen, T. Andreadis, S.M. Anlage, Acta Phys. Pol. A 124, 1045 (2013).

[7] X. Zheng, S. Hemmady, T.M. Antonsen, Jr.S.M. Anlage, E. Ott, Phys.Phys. Rev. E 73046208 (2006).

[8] H.-J. Stöckmann, J. Stein, Phys. Rev. Lett. 64, 2215 (1990).

[9] S. Sridhar, A. Kudrolli, Phys. Rev. Lett. 72, 2175 (1994).

[10] L. Sirko, P.M. Koch, R. Blümel, Phys. Rev. Lett. 78, 2940 (1997).

[11] Y. Hlushchuk, A. Kohler, S. Bauch, L. Sirko, R. Blümel, M. Barth, H.-J. Stöckmann, Phys. Rev. E 61, 366 (2000).

[12] Y. Hlushchuk, A. Błędowski, N. Savytskyy, L. Sirko, Phys. Scr. 64, 192 (2001).

[13] Y. Hlushchuk, L. Sirko, U. Kuhl, M. Barth, H.-J. Stöckmann, Phys. Rev. E 63, 046208 (2001).

[14] R. Blümel, P.M. Koch, L. Sirko, Found. Phys. 31, 269 (2001).

[15] A. Dhar, D. M. Rao, U. Shankar, S. Sridhar, Phys. Rev. E 68, 026208 (2003).

[16] N. Savytskyy, O. Hul, L. Sirko, Phys. Rev. E 70, 056209 (2004).

[17] S. Hemmady, X. Zheng, E. Ott, T.M. Antonsen, S.M. Anlage, Phys. Rev. Lett. 94, 014102 (2005).

[18] O. Hul, N. Savytskyy, O. Tymoshchuk, S. Bauch, L. Sirko, Phys. Rev. E 72, 066212 (2005).

[19] B. Dietz, A. Richter, CHAOS 25, 097601 (2015).

[20] M. Białous, B. Dietz, L. Sirko, Phys. Rev. E 100, 012210 (2019).

[21] B. Dietz, T. Klaus, M. Miski-Oglu, A. Richter, M. Wunderle, Phys. Rev. Lett. 123, 174101 (2019).

[22] T. Zimmermann, H. Köppel, L.S. Cederbaum, G. Persch, W. Demtröder, Phys. Rev. Lett. 61, 3 (1988).

[23] R. Blümel, A. Buchleitner, R. Graham, L. Sirko, U. Smilansky, H. Walther, Phys. Rev. A 44, 4521 (1991).

[24] R.V. Jensen, S.M. Susskind, M.M. Sanders, Physics Reports 201, 1 (1991). 
[25] M. Bellermann, T. Bergemann, A. Haffmann, P.M. Koch, L. Sirko, Phys. Rev. A 46, 5836 (1992).

[26] L. Sirko, S. Yoakum, A. Haffmans, P.M. Koch, Phys. Rev. A 47, R782 (1993).

[27] A. Buchleitner, D. Delande, Phys. Rev. Lett. 71, 3633 (1993).

[28] L. Sirko, M.R.W. Bellermann, A. Haffmans, P.M. Koch, D. Richards, Phys. Rev. Lett. 71, 2895 (1993).

[29] J.E. Bayfield, S.-Y. Luie, L.C. Perotti, M.P. Skrzypkowski, Physica D: Nonlinear Phenomena 83, 46 (1995).

[30] L. Sirko, P.M. Koch, Appl. Phys. B 60, S195 (1995).

[31] L. Sirko, A. Haffmans, M.R.W. Bellermann, P.M. Koch, Europhys. Lett. 33, 181 (1996).

[32] J. Bayfield, Lal Pinnaduwage, J. Phys. B 18, L49 (1999).

[33] L. Sirko, S.A. Zelazny, P.M. Koch, Phys. Rev. Lett. 87, 043002 (2001).

[34] L. Sirko, P.M. Koch, Phys. Rev. Lett. 89, 274101 (2002).

[35] A. Arakelyan, J. Nunkaew, T.F. Gallagher, Phys. Rev. A 94, 053416 (2016).

[36] L. Vina, M. Potemski, W. Wang, Phys.Ups. 41, 153 (1998).

[37] O. Hul, S. Bauch, P. Pakoński, N. Savytskyy, K. Życzkowski, L. Sirko, Phys. Rev. E 69, 056205 (2004).

[38] O. Hul, O. Tymoshchuk, S. Bauch, P.M. Koch, L. Sirko, J. Phys. A 38, 10489 (2005).

[39] O. Hul, M. Ławniczak, S. Bauch, A. Sawicki, M. Kuś, L. Sirko, Phys. Rev. Lett 109, 040402 (2012).

[40] B. Dietz, V. Yunko. M. Białous, S. Bauch, M. Ławniczak, L. Sirko, Phys. Rev. E 95, 052202 (2017).

[41] M. Ławniczak, J. Lipovský, L. Sirko, Phys. Rev. Lett. 122, 140503 (2019).

[42] M. Ławniczak, J. Lipovský, M. Białous, L. Sirko, Phys. Rev. E 103, 032208 (2021).

[43] M. Ławniczak, A. Sawicki, M. Białous, L. Sirko, Sci. Rep. 11, 1575 (2021).

[44] M. Ławniczak, P. Kurasov, S. Bauch, M. Białous, A. Akhshani, L. Sirko, Sci. Rep. 11, 15342 (2021).

[45] J.B. French, V.K.B. Kota, A. Pandey, S. Tomsovic, Phys. Rev. Lett. 54, 2313 (1985).

[46] G.E. Mitchell, A. Richter, H.A. Weidenmüller, Rev. Mod. Phys. 82, 2845 (2010).

[47] K. Sacha, J. Zakrzewski, D. Delande, Phys. Rev. Lett. 83, 2922 (1999).
[48] L.A. Ponomarenko, F. Schedlin, M.I. Katsnelson, R. Yang, E.W. Hill, K.S. Novoselov, A.K. Geim, Science 320, 356 (2008).

[49] M. Aßmann, J. Thewes, D. Fröhlich, M. Bayer, Nat. Mater. 15, 741 (2016).

[50] P. So, S. M. Anlage, E. Ott, R.N. Oerter, Phys. Rev. Lett. 74, 2662 (1995).

[51] U. Stoffregen, J. Stein, H.-J. Stöckmann, M. Kuś, F. Haake, Phys. Rev. Lett. 74, 2666 (1995).

[52] M. Ławniczak, S. Bauch, O. Hul, L. Sirko, Phys. Rev. E 81, 046204 (2010).

[53] M. Białous, V. Yunko, S. Bauch, M. Ławniczak, B. Dietz, L. Sirko, Phys. Rev. Lett. 117, 144101 (2016).

[54] M. Ławniczak L. Sirko, Sci. Rep. 9, 5630 (2019).

[55] J. Lu, J. Che, X. Zhang, B. Dietz, Phys. Rev. E 102, 022309 (2020).

[56] V. Yunko, M. Białous, L. Sirko, Phys. Rev. E 102, 012210 (2020).

[57] A. Pandey, P. Shukla, J. Phys. A 24, 3907 (1991).

[58] G. Lenz, K. Życzkowski, J. Phys. A 25, 5539 (1992).

[59] O. Bohigas, M.-J. Giannoni, A.M.O. de Almeidaz, C. Schmit, Nonlinearity 8, 203 (1995).

[60] B. Dietz, T. Friedrich, H.L. Harney, M. Miski-Oglu, A. Richter, F. Schäfer, J. Verbaarschot, H.A. Weidenmüller, Phys. Rev. Lett. 103, 064101 (2009).

[61] S. Schierenberg, F. Bruckmann, T. Wetting, Phys. Rev. E 85, 061130 (2012).

[62] M. Białous, B. Dietz, L. Sirko,Phys. Rev. E 103, 052204 (2021).

[63] M. Białous, B. Dietz, L. Sirko, Phys. Rev. E 102, 042206 (2020).

[64] M. Białous, B. Dietz, L. Sirko, Acta Phys. Pol. A 139, 462 (2021).

[65] R.A. Molina, J. Retamosa, L. Muñoz, A. Relaño, E. Faleiro, Phys. Lett. B 644, 25 (2007).

[66] O. Bohigas, M.P. Pato, Phys. Lett. B 595, 171 (2004).

[67] M. Białous, B. Dietz, L. Sirko, Phys. Rev. E 100, 012210 (2019).

[68] A. Relaño, J.M.G. Gómez, R.A. Molina, J. Retamosa, E. Faleiro, Phys. Rev. Lett. 89, 244102 (2002).

[69] E. Faleiro, J.M.G. Gómez, R.A. Molina, L. Muñoz, A. Relaño, J. Retamosa, Phys. Rev. Lett. 93, 244101 (2004). 